Résumés des conférences et travaux

\title{
Histoire de l'art du Moyen Âge occidental
}

\section{Histoire de l'art du Moyen Âge occidental}

Conférences de l'année 2013-2014

Philippe Lorentz

\section{OpenEdition \\ Journals}

Édition électronique

URL : https://journals.openedition.org/ashp/1727

DOI : 10.4000/ashp.1727

ISSN : 1969-6310

Éditeur

Publications de l'École Pratique des Hautes Études

Édition imprimée

Date de publication : 1 septembre 2015

Pagination : 191-196

ISSN : 0766-0677

Référence électronique

Philippe Lorentz, "Histoire de l'art du Moyen Âge occidental », Annuaire de l'École pratique des hautes études (EPHE), Section des sciences historiques et philologiques [En ligne], 146 | 2015, mis en ligne le 02 octobre 2015, consulté le 09 juillet 2021. URL : http://journals.openedition.org/ashp/1727 ; DOI : https://doi.org/10.4000/ashp.1727 


\title{
HISTOIRE DE L'ART DU MOYEN ÂGE OCCIDENTAL
}

\author{
Directeur d'études : M. Philippe LoREnTZ
}

Programme de l'année 2013-2014 : La peinture à Paris au XVe siècle : la famille d'Ypres.

Une partie de l'activité des peintres et des enlumineurs dans ce grand foyer de création qu'a été Paris dans la seconde moitié du $\mathrm{Xv}^{\mathrm{e}}$ siècle a fait l'objet des conférences de l'année 2013-2014. L'étude de la production artistique dans la capitale du royaume de France au cours des décennies qui ont suivi l'occupation anglaise méritait une attention renouvelée, suscitée par des travaux récents. En exploitant systématiquement les documents du Minutier central des notaires parisiens avant 1515, Étienne Hamon a établi un dictionnaire d'artistes, accompagné d'un choix de documents analysés ${ }^{1}$. Ce recueil de sources, qui concerne aussi bien les métiers du bâtiment que ceux du mobilier ou encore les peintres, enlumineurs, verriers, sculpteurs et tapissiers, a révélé de nombreux noms d'artistes / artisans, dans le sillage de la publication de Guy-Michel Leproux pour la période suivante ${ }^{2}$. Mis à la disposition de l'historien, ce matériau documentaire a en grande partie nourri la synthèse proposée par Étienne Hamon sur l'architecture à Paris sous Charles VIII et Louis XII, issue du mémoire inédit de son habilitation à diriger des recherches ${ }^{3}$. Centrée sur l'architecture, cette étude fait également une place aux arts figurés au service du décor monumental. Dans son volume de Documents du Minutier central des notaires de Paris, l'auteur fait une mise au point sur les membres de la famille d'Ypres, dont plusieurs membres ont exercé le métier de peintre et d'enlumineur à Paris du milieu du $X^{\mathrm{e}}$ siècle au début du siècle suivant, notamment Nicolas d'Ypres, également connu sous le nom de Colin d'Amiens, probablement le Maître de Coëtivy, et ses fils Jean et Louis d'Ypres, dont l'activité correspond sans doute au nom de convention de Maître des Très Petites Heures d'Anne de Bretagne (ou Maître de la Rose de la Sainte-Chapelle). Ina Nettekoven a publié en 2004 un ouvrage sur le Maître de la Rose de la Sainte-Chapelle, auquel elle a consacré sa thèse de doctorat ${ }^{4}$. Ce (ou ces) peintre(s) a (ou ont) été à la tête de l'un des principaux ateliers parisiens à la fin $\mathrm{du} \mathrm{XV}^{\mathrm{e}}$ et au début du $\mathrm{XVI}^{\mathrm{e}}$ siècle. Ces recherches récentes ont été propices à un examen à nouveaux frais du dossier de la famille d'Ypres, à commencer par le fondateur de la dynastie : André d'Ypres (le Maître de Dreux Budé ?), originaire d'Amiens, qui s'établit vers 1445 à Paris.

1. Étienne Hamon, Minutier central des notaires de Paris. Art et architecture avant 1515, Paris, 2008.

2. Guy-Michel Leproux, La peinture à Paris sous le règne de François I Ir , Paris, 2001 (Corpus Vitrearum, France, Études, IV).

3. Soutenue en 2008 (garant: Dany Sandron). Voir Étienne Hamon, Une capitale flamboyante. La création monumentale à Paris autour de 1500, Paris, 2011.

4. Ina Nettekoven, Der Meister der Apocalypsenrose der Sainte-Chapelle und die Pariser Buchkunst um 1500, Turnhout, 2004. 
La reprise de la commande artistique à la fin de la guerre de Cent Ans met en évidence l'adhésion précoce de certains notables parisiens aux nouveautés picturales élaborées dans les anciens Pays-Bas. L'ars nova flamande fait une brusque irruption dans la capitale dès le retour à la sécurité instauré par les trêves de Tours (1444). Cette arrivée de formes nouvelles s'implante durablement, sans toutefois que les Parisiens qui commandent retables et manuscrits enluminés n'abandonnent l'esthétique en vigueur au cours des deux premières décennies du $\mathrm{Xv}^{\mathrm{e}}$ siècle, à laquelle ils restent fidèles jusque dans les années 1460. Dans le Paris anglais, la production de manuscrits enluminés, certes très ralentie par rapport à la période précédente, est restée brillante, comme le donne à voir l'œuvre du Maître de Bedford, dont le " principal associé », le Maître de Dunois, continue à être sollicité par de nombreux commanditaires jusqu'au milieu du $\mathrm{Xv}^{\mathrm{e}}$ siècle. Cet artiste est l'auteur d'un retable de la Trinité exécuté pour les chanoines de la cathédrale Notre-Dame (vers 1440 ? Paris, École nationale supérieure des beaux-arts), étudié sur son lieu de conservation lors d'une séance du séminaire en janvier 2014.

Peu avant 1450, on assiste à l'arrivée dans la capitale d'un peintre apportant dans ses bagages une toute autre culture, familier du répertoire du Maître de Flémalle et de Rogier van der Weyden. Son art séduit les élites. Le Parlement lui passe commande d'une Crucifixion pour sa Grande Chambre ; un haut fonctionnaire royal, Dreux Budé, lui fait faire un retable pour sa chapelle familiale en l'église Saint-Gervais. La chronologie de ces œuvres a été réexaminée de manière approfondie. Restée en place depuis sa création jusqu'en 1796, la Crucifixion du Parlement de Paris a été commentée dès la seconde moitié du XVIII ${ }^{\mathrm{e}}$ siècle. L'abondante littérature regardant son attribution et l'époque de sa réalisation a été passée en revue. La Crucifixion a souvent été située, par le passé, au cours des dernières décennies du $\mathrm{XV}^{\mathrm{e}}$ siècle et ce n'est que récemment qu'une date autour de 1450 s'est imposée au prix d'une enquête historique portant sur les circonstances de la commande et sur le mode de financement du tableau ${ }^{1}$. La collecte des fonds destinés à la nouvelle Crucifixion a vraisemblablement commencé avant 1449. La somme que la documentation conservée nous donne à connaître s'élève à environ 300 livres parisis. Elle est confiée par le Parlement à l'un de ses conseillers, Jehan Paillart, également chanoine de Notre-Dame, probablement chargé du choix du peintre, des négociations avec ce dernier et du suivi de la réalisation du tableau. Après sa mort, survenue entre janvier et avril 1454, le Parlement récupère de ses héritiers et exécuteurs testamentaires le reliquat de cet argent, soit 143 livres parisis, qui doivent correspondre au dernier acompte en attente d'être versé à l'artiste lors de la réception de la Crucifixion. Le coût élevé d'un ouvrage de cette importance explique un financement étalé sur plusieurs années et il n'y a rien d'étonnant à ce que l'entreprise, menée à son terme en 1453, ait débuté en 1449, voire auparavant.

Les registres du Parlement fournissent des données chronologiques et pécuniaires sur la Crucifixion de la Grande Chambre. Ils ne donnent pas le nom du peintre, car la cour souveraine a délégué à Jehan Paillart la gestion du budget alloué au tableau. La tâche de l'historien de l'art ne se limite pas aux recherches documentaires et c'est

1. Philippe Lorentz, «La Crucifixion du Parlement de Paris », dans P. Lorentz et Micheline ComblenSonkes, Musée du Louvre. Paris, t. III, Bruxelles, 2001 (Corpus de la peinture des anciens Pays-Bas méridionaux au quinzième siècle, 19), p. 81-132. 
l'analyse du style de cette œuvre qui nous en apprend davantage sur son auteur. Elle nous indique qu'il a fréquenté Tournai, l'un des principaux foyers des anciens PaysBas méridionaux. Il a en effet emprunté un certain nombre de ses figures au Maître de Flémalle et à Rogier van der Weyden, les principaux acteurs du renouveau pictural dans cette cité. Comme eux, il s'attache à rendre la surface des objets et la texture des matières. Longtemps isolée, la Crucifixion du Parlement fait désormais partie d'un petit groupe d'œuvres (tableaux, enluminures et vitraux), dont l'ancrage à Paris au milieu du $\mathrm{Xv}^{\mathrm{e}}$ siècle prouve une implantation durable dans la capitale de ce peintre formé dans les anciens Pays-Bas. Un triptyque de la Crucifixion peint pour Dreux Budé, notaire et secrétaire du roi, lui a valu l'appellation de « Maître de Dreux Budé. » Son activité parisienne touche à la production de manuscrits enluminés. Ses miniatures sont si proches, par le style, de celles d'un peintre et enlumineur lui aussi actif à Paris dans la seconde moitié du Xv $\mathrm{Vv}^{\mathrm{e}}$ siècle, le Maître de Coëtivy, qu'un certain nombre d'entre elles ont été autrefois attribuées à ce dernier, plus jeune d'une génération. Cette parenté stylistique peut être élucidée par l'existence de réels liens de parenté ayant uni les deux artistes, l'un étant le fils de l'autre. Le Maître de Dreux Budé pourrait être André d'Ypres, un peintre connu à Amiens depuis 1425-1426, devenu franc-maître à Tournai en 1428, à l'époque même où Rogier van der Weyden travaille dans l'atelier de Robert Campin ${ }^{1}$. Il s'établit à Paris après 1444 et décède à Mons en juillet 1450, lors du retour d'un pèlerinage à Rome ${ }^{2}$. Un acte notarié de 1479 nous apprend qu'il est le père de Nicolas (ou « Colin ») d'Ypres, dit « d'Amiens » ${ }^{3}$. Ce dernier serait donc le Maître de Coëtivy, une identification corroborée par la découverte d'une œuvre documentée conçue par Nicolas d'Amiens, une Mise au tombeau sculptée en 1495-1496 d'après le patron fait par cet artiste, destinée à la chapelle du château de l'amiral Louis Malet de Graville, à Malesherbes (Loiret) ${ }^{4}$. De fait, un certain nombre de parallèles peuvent être tracés entre les figures de ce groupe sculpté et celles de la Résurrection de Lazare, panneau du Maître de Coëtivy conservé au Louvre ${ }^{5}$.

À la fin du Xv $\mathrm{Xv}^{\mathrm{e}}$ et au début du $\mathrm{XVI}^{\mathrm{e}}$ siècle, un atelier a occupé le devant de la scène artistique parisienne en faisant fructifier le savoir-faire et les modèles accumulés au fil du temps par le Maître de Dreux Budé et le Maître de Coëtivy. Le (ou les) artiste(s) dirigeant cette officine ont fourni des patrons pour des supports variés, ce qui leur a valu plusieurs noms de convention, comme ceux de Maître de la Rose de la SainteChapelle ou de Maître de la Vie de saint Jean-Baptiste pour le vitrail, de Maître de la

1. Nicole Reynaud est la première à avoir formulé cette hypothèse en 1993, renonçant à son identification du Maître de Coëtivy avec le Néerlandais Henri de Vulcop, peintre de la reine Marie d'Anjou (N. Reynaud, dans François Avril et N. Reynaud, catalogue de l'exposition Les manuscrits à peintures en France. 1440-1520, Paris, Bibliothèque nationale, 1993, p. 53).

2. Dominique Vanwijnsberghe, «Du nouveau sur le peintre André d'Ypres, artiste du Nord installé à Paris », dans Bulletin monumental, 158 (2000), p. 365-369.

3. Henri Martin, «Les d'Ypres, peintres des $\mathrm{Xv}^{\mathrm{e}}$ et $\mathrm{XvI}^{\mathrm{e}}$ siècles », Archives de l'Art français, nouvelle période, 8 (1916), p. 10-11.

4. Catherine Grodecki, « Le "maître Nicolas d'Amiens" et la mise au tombeau de Malesherbes », Bulletin monumental, 1996, p. 329-342.

5. P. Lorentz, « The Painter's Role in the Conception of Sculpture: Jean Hey at Chantelle », dans Julien Chapuis (dir.), Invention. Northern Renaissance Studies in Honor of Molly Faries, Turnhout, 2008, p. 127-129, fig. 3-7. 
Chasse à la Licorne pour la tapisserie et de Maître des Très Petites Heures d'Anne de Bretagne pour l'enluminure. Or la famille d'Ypres, peintres du nord ayant fait souche à Paris, compte une troisième génération, représentée dans la capitale par deux fils de Nicolas d'Amiens : Jean d'Ypres († 1508), élu juré du métier des peintres à Paris en 1504, et son frère Louis, peintre lui aussi ${ }^{1}$. Ces deux artistes sont évidemment de solides candidats à l'identification avec le Maître des Très Petites Heures d'Anne de Bretagne. Cette lignée de peintres nous met en présence d'une de ces structures familiales si fréquemment rencontrées dans le monde artisanal de la fin du Moyen Âge. Grâce à un faisceau de présomptions, il est donc possible de mettre en parallèle le témoignage des œuvres, reflétant l'activité d'une triade d'artistes qui se sont succédés à Paris durant la seconde moitié du XV $\mathrm{Xv}^{\mathrm{e}}$ siècle et les premières années du $\mathrm{XVI}^{\mathrm{e}}$ siècle, avec la présence documentée de la famille d'Ypres dont on suit la trace dans la capitale depuis la fin des années 1440.

L'hypothèse de la famille d'Ypres a été remise en cause par Ina Nettekoven dans sa thèse de doctorat consacrée au Maître de la Rose de la Sainte-Chapelle ${ }^{2}$. Les réserves qu'elle exprime se rapportent pour l'essentiel au représentant de la première génération, le Maître de Dreux Budé, et au problème posé par l'existence de mentions relatives au paiement de la Crucifixion du Parlement postérieures à la mort du peintre André d'Ypres (1450), preuve selon elle que ce dernier ne peut être l'auteur du grand tableau. La lecture contextuelle du dossier documentaire dont les principaux éléments ont été rappelés plus haut - fonds collectés à partir de 1449, voire avant; dernière somme à acquitter disponible en 1454 -, ne peut cependant conduire à la même conclusion. L'exécution d'une œuvre de cette ampleur est une entreprise de longue haleine : les sources d'archives $\mathrm{du} \mathrm{Xv}^{\mathrm{e}}$ siècle fournissent de nombreux témoignages de grands retables achevés hors délais, plusieurs années après leur mise en chantier. Le Parlement ne disposant pas de la somme totale lorsque l'ouvrage a débuté, l'argent a été injecté dans l'opération de manière progressive, ce qui explique la lenteur de l'exécution.

La Crucifixion n'est pas complètement payée en 1454, soit près de quatre ans après le décès à Mons d' ' Andrieu d'Ippre, peintre de Paris », en juillet 1450, lors de son retour de Rome, où il vient d'accomplir le pèlerinage de l'année jubilaire. Mais le tableau est déjà en chantier en février 1449, un an et demi avant la disparition du peintre. Pour Ina Nettekoven, il n'est pas envisageable qu'un artiste soucieux de maintenir sa bonne renommée ait pu partir pour un voyage long et périlleux en confiant à ses collaborateurs l'achèvement d'une commande aussi importante que le tableau du Parlement. Que dire alors de Rogier van der Weyden, qui se rend lui aussi à Rome en 1450 en laissant dans son atelier le Polyptyque du Jugement dernier, destiné à un client aussi éminent que le chancelier Nicolas Rolin? En prenant la décision de prendre part aux célébrations de l'année sainte, André d'Ypres et Rogier van der Weyden n'abandonnent pas à leurs ateliers respectifs l'achèvement des chantiers en cours. L'un et l'autre comptent bien revenir après le pèlerinage pour en superviser les opérations finales. Au milieu du $\mathrm{Xv}^{\mathrm{e}}$ siècle, un voyage à Rome n'est certes pas le déplacement anodin que nous pratiquons aujourd'hui, mais n'a rien d'exceptionnel et ceux

1. G.-M. Leproux, 2001, op. cit., p. 57 ; E. Hamon, 2008, op. cit., $\mathrm{n}^{\circ} 954$.

2. I. Nettekoven, 2004, op. cit., p. 31-37. 
qui l'entreprennent - André d'Ypres inclus - ont le ferme espoir de revenir vivants ! Les affinités stylistiques entre l'œuvre du Maître de Dreux Budé et celle du Maître de Coëtivy accréditent l'identification de ce dernier avec Nicolas d'Amiens, fils d'André d'Ypres et peintre lui aussi, qui a probablement assumé les dernières étapes de l'exécution du « tableau de la grant chambre de parlement » après la mort de son père. En de telles circonstances, l'avenir de l'atelier familial était en jeu et il n'y a pas à s'étonner de voir le fils assumer une commande laissée inachevée par son père.

Le peintre du tableau du Parlement (ou Maître de Dreux Budé) est également l'auteur d'un Triptyque de la Crucifixion réalisé pour Dreux Budé, audiencier de la chancellerie royale, puis garde des chartes du roi, et son épouse Jeanne Peschard ${ }^{1}$. Ces deux personnages figurent à genoux sur les volets, tournés vers la Crucifixion du panneau central (Los Angeles, J. Paul Getty Museum). Les volets représentent respectivement l'Arrestation du Christ (volet gauche ; collection privée) et la Résurrection (volet droit ; Montpellier, musée Fabre). La largeur du panneau central $(71,5 \mathrm{~cm})$ correspond aux dimensions d'un autel de chapelle. Depuis son installation à Paris, la famille Budé est implantée dans la paroisse Saint-Gervais-Saint-Protais. Au milieu du $\mathrm{Xv}^{\mathrm{e}}$ siècle, Dreux Budé et Jeanne Peschard fondent, dans le chœur de l'église une chapelle consacrée à la Vierge et à saint Christophe. La présence de saint Christophe introduisant Dreux Budé sur le volet gauche du Triptyque de la Crucifixion ne laisse subsister aucun doute quant à l'appartenance originelle de ce retable au mobilier liturgique de la chapelle Budé à Saint-Gervais. Dans son histoire de cette paroisse parisienne, publiée en 1950, le chanoine Louis Brochard nous apprend que Dreux Budé et Jeanne Peschard ont fait construire leur chapelle en 1454. Charles Sterling, qui se fonde sur cet auteur, a retenu la date comme celle de l'exécution du triptyque ${ }^{2}$. Reprenant ces données, Ina Nettekoven avance que les Budé passent commande pour la construction de la chapelle en $1454^{3}$. Elle considère que le retable aurait été commandé à la même époque, ce qui écarte formellement André d'Ypres comme peintre du retable, puisque celui-ci est mort en 1450. Le recours aux sources incite à davantage de prudence.

Aucun document ne stipule que Dreux Budé et Jeanne Peschard font élever leur chapelle en 1454. En revanche, dans ce qui subsiste des archives de la paroisse, est conservé un acte important conclu le 26 mai 1453 entre le couple et les fabriciens de l'église 4 . Il contient toutes les dispositions prévues par Dreux Budé et son épouse pour la célébration d'une messe quotidienne et de quatre obits annuels pour le salut de leurs âmes et des membres de leur famille. Il est prévu que ces messes et services seront dits en la chapelle que le couple a « de nouvel» fait construire. En 1453, la chapelle Budé n'est pas à l'état de projet, mais existe bel et bien. Située au chevet de Saint-Gervais, elle disparaîtra quelques décennies plus tard, lors de la reconstruction du chœur de

1. Le triptyque a été reconstitué par Burton Fredericksen, «A Parisian Triptych Reconstituted», The J. Paul Getty Museum Journal, 11 (1983), p. 183-196. Pour l'identification des donateurs, voir Charles Sterling, La Peinture médiévale à Paris. 1300-1500, 2, Paris, 1990, p. 50-75 ; P. Lorentz, "Triptych of Dreux Budé », dans Martha Wolff (dir.), Kings, Queens and Courtiers. Art in Early Renaissance France, New Haven et Londres, 2011, p. 52-55, $\mathrm{n}^{\text {os }}$ 6-8.

2. Louis Brochard, Saint-Gervais. Histoire de la paroisse d'après de nombreux documents inédits, Paris, 1950, p. 119 ; C. Sterling, 1990, op. cit., p. 58-59.

3. I. Nettekoven, 2004, op. cit., p. 35.

4. Paris, Archives nationales, L 651. 
l'église à partir de 1494. En 1453, elle est édifiée « de nouvel », un terme peu précis, qui peut aussi bien renvoyer à quelques mois ou à quelques années. En somme, lorsque les Budé procèdent à leur fondation de messes, leur chapelle familiale est peut-être en état de fonctionnement depuis déjà plusieurs années. En l'état actuel de nos connaissances, force est de constater que la question de la date du Triptyque de la Crucifixion, peint pour Dreux Budé et Jeanne Peschard, reste ouverte. Nous ne disposons pas de preuve selon laquelle ce retable aurait été réalisé avant 1450. Mais, tel que le dossier se présente à nous aujourd'hui, rien ne permet non plus de le situer après cette date. André d'Ypres reste donc un candidat sérieux pour une identification avec le Maître de Dreux Budé, de même que son petit-fils Jean d'Ypres pourrait bien être le Maître des Très Petites Heures d'Anne de Bretagne, alias le Maître de la Rose de la SainteChapelle. On ne peut pas non plus suivre Ina Nettekoven lorsqu'elle écarte le représentant de la troisième génération de cette famille de peintres. Selon elle, Jean d'Ypres ne serait devenu maître qu'en 1504 et il n'est pas pensable que l'on ait fait appel à un artiste dépourvu de ce titre pour une commande de l'importance de la nouvelle rose de la Sainte-Chapelle, dont les travaux commencent vers 1485-1490. Or en 1504, Jean d'Ypres n'accède pas à la maîtrise, mais est élu juré de la corporation des peintres et sculpteurs de Paris, qui comportait quatre jurés, élus pour deux ans et renouvelés chaque année par moitié ${ }^{1}$. Qu'André d'Ypres, Nicolas d'Amiens et Jean d'Ypres soient le Maître de Dreux Budé, le Maître de Coëtivy et le Maître des Très Petites Heures d'Anne de Bretagne reste bien entendu une hypothèse, en attente d'être confirmée ou infirmée par la découverte de nouveaux documents, mais non au prix d'une distorsion des témoignages historiques. 